\title{
Metabolic Activation of the Tumorigenic Pyrrolizidine Alkaloid, Retrorsine, Leading to DNA Adduct Formation In Vivo
}

\author{
Yu-Ping Wang ${ }^{1 *}$, Peter P. Fu ${ }^{1}$, and Ming W. Chou ${ }^{1}$ \\ ${ }^{1}$ National Center for Toxicological Research, Jefferson, AR 72079 \\ *Correspondence to Dr. Yu-Ping Wang. E-mail: ywang@nctr.fda.gov
}

Received: 15 November 2004 / Accepted: 06 February 2005 / Published: 30 April 2005

\begin{abstract}
Pyrrolizidine alkaloids are naturally occurring genotoxic chemicals produced by a large number of plants. The high toxicity of many pyrrolizidine alkaloids has caused considerable loss of free-ranging livestock due to liver and pulmonary lesions. Chronic exposure of toxic pyrrolizidine alkaloids to laboratory animals induces cancer. This investigation studies the metabolic activation of retrorsine, a representative naturally occurring tumorigenic pyrrolizidine alkaloid, and shows that a genotoxic mechanism is correlated to the tumorigenicity of retrorsine. Metabolism of retrorsine by liver microsomes of F344 female rats produced two metabolites, 6, 7-dihydro-7-hydroxy-1-hydroxymethyl-5H-pyrrolizine (DHP), at a rate of $4.8 \pm 0.1 \mathrm{nmol} / \mathrm{mg} / \mathrm{min}$, and retrorsine-N-oxide, at a rate of $17.6 \pm 0.5 \mathrm{nmol} / \mathrm{mg} / \mathrm{min}$. Metabolism was enhanced 1.7-fold by using liver microsomes prepared from dexamethasone-treated rats. DHP formation was inhibited $77 \%$ and retrorsine $\mathrm{N}$-oxide formation was inhibited $29 \%$ by troleandomycin, a P450 3A enzyme inhibitor. Metabolism of retrorsine with lung, kidney, and spleen microsomes from dexamethasone-treated rats also generated DHP and the N-oxide derivative. When rat liver microsomal metabolism of retrorsine occurred in the presence of calf thymus DNA, a set of DHP-derived DNA adducts was formed; these adducts were detected and quantified by using a previously developed ${ }^{32} \mathrm{P}$-postlabeling/HPLC method. These same DNA adducts were also found in liver DNA of rats gavaged with retrorsine. Since DHP-derived DNA adducts are suggested to be potential biomarkers of riddelliineinduced tumorigenicity, our results indicate that (i) similar to the metabolic activation of riddelliine, the mechanism of retrorsine-induced carcinogenicity in rats is also through a genotoxic mechanism involving DHP; and (ii) the set of DHP-derived DNA adducts found in liver DNA of rats gavaged with retrorsine or riddelliine can serve as biomarkers for the tumorigenicity induced by retronecine-type pyrrolizidine alkaloids.
\end{abstract}

Keywords: pyrrolizidine alkaloids, retrorsine, riddelliine, 6, 7-dihydro-7-hydroxy-1-hydroxymethyl-5Hpyrrolizine (DHP), DHP-derived DNA adduct, ${ }^{32}$ P-postlabeling/HPLC, Troleandomycine (TAO).

\section{Introduction}

Pyrrolizidine alkaloids are common constituents of hundreds of plant species of different unrelated botanical families distributed in many geographical regions in the world (1-10). It has been reported that about $3 \%$ of the world's flowering plants contain pyrrolizidine alkaloids (11). Pyrrolizidine alkaloids share a common chemical structure that consists of a necine-base and a necic acid moiety. The alkaloids with the necine base containing an unsaturated double bond at 1, 2-position, such as retronecine, heliotridine, and otonecine are the most toxic pyrrolizidine alkaloids. Among these, the retronecine-based pyrrolizidine alkaloids are abundant and their toxicities have been studied more extensively. Pyrrolizidine alkaloid per se is not toxic. Metabolic activation is required to form a pyrrolic metabolite to exert their toxicity. The high toxicity of many of pyrrolizidine alkaloid-containing plants has caused great loss of free-ranging livestock due to liver and pulmonary lesions. In addition, chronic exposure of toxic pyrrolizidine alkaloids to laboratory rats and mice induces tumors. Because of the concern of human exposure to genotoxic pyrrolizidine alkaloids, toxicity and carcinogenicity of riddelliine, a representative pyrrolizidine alkaloid, have been studied by the National Toxicology Program (NTP) conducted by National Institute of Environmental Health Sciences (NIEHS) (12); and the mechanism of the riddelliine-induced tumorigenicity in rats and mice was studied at the National Center for Toxicological Research (NCTR) (13-16). Results of the mechanistic study showed that 
riddelliine was metabolized to form a reactive pyrrolic metabolite, 6, 7-dihydro-7-hydroxy-1-hydroxymethyl$5 \mathrm{H}$-pyrrolizine (DHP), as well as riddelliine $\mathrm{N}$-oxide (13). DHP bound to DNA in vitro and in vivo generated DHP-derived DNA adducts that were determined by the ${ }^{32}$ P-postlabeling/ HPLC analyses $(14,15)$. The levels of these DNA adduct formations correlate with the liver tumor potency of riddelliine (16) implying that the riddelliine-induced carcinogenesis is through a genotoxic mechanism.

Retrorsine is another representative retronecine-based pyrrolizidine alkaloid. Similar to riddelliine, retrorsine is a 12-membered macrocyclic diester pyrrolizidine alkaloid with an $\alpha, \beta$-unsaturated double bond linked to the ester group at $\mathrm{C}-7$ position of the retronecine base. The toxicity of retrorsine is due to the metabolic formation of the reactive pyrrolic metabolite $(17,18)$; however, the mechanism of retrorsine-induced tumorigenicity is not clear. Since retrorsine is structurally similar to that of riddelliine, it is important to know whether the genotoxic mechanism of riddelliineinduced tumorigenicity is also that of retrorsine. In this paper, we report results of the study on the metabolic activation of retrorsine. The detection of DHP-derived DNA adducts formation in liver DNA of rats treated with retrorsine indicates that the tumorigenicity of retrorsine is also through a genotoxic mechanism.

\section{Materials and Methods}

\section{Materials}

Retrorsine, retrorsine N-oxide and troleandomycin (triacetyloleandomycin, TAO) were purchased from Sigma Chemical Co. (St. Louis, MO). $\left[\gamma-{ }^{32} \mathrm{P}\right]$ Adenosine 5'- triphosphate ( $\left[{ }^{32} \mathrm{P}\right]$ ATP) (sp. Act. $>7,000 \mathrm{Ci} / \mathrm{mmol}$ ) was purchased from ICN Biomedicals, Inc. (Costa Mesa, $\mathrm{CA})$. Enzymes required for DNA hydrolysis and for ${ }^{32} \mathrm{P}-$ postlabeling/HPLC analysis were purchased and used as previously described (14). DHP and the 3'monophosphate of 7-(deoxyguanosine- $\mathrm{N}^{2}$-yl) dehydrosupinidine adducts (DHP-3'-dGMP) were prepared in our laboratory $(14,18)$. The liver microsomes of untreated rats (control microsomes) were prepared as previously described (13) and liver microsomes of female F344 rats pretreated with dexamthesasone (dosed daily with $75 \mathrm{mg}$ dexamethasone $/ \mathrm{kg}$ body weight intraperitoneally, for three consecutive days) were prepared similarly. Microsomes from rat lung, kidney, and spleen were also prepared similarly.

\section{Animals and Treatment}

Female F344 rats (3 per group) were obtained from the NCTR breeding colony as weanlings and maintained on a $12 \mathrm{~h}$ light-dark cycle. At 8 weeks of age, three animals/ treatment were dosed by oral gavages with retrorsine at $1.0 \mathrm{mg} / \mathrm{kg}$ /day in $0.1 \mathrm{M}$ phosphate buffer $(\mathrm{pH}, 8.0)$ for three consecutive days. Control animals were gavaged with $0.1 \mathrm{M}$ phosphate buffer only. Twenty-four hours after final dosing, the animals were euthanized by exposure to carbon dioxide and liver tissues were excised and stored at $-80{ }^{\circ} \mathrm{C}$.

\section{In vitro Metabolism of Retrorsine}

Metabolism of retrorsine by control or dexamethasone-induced rat liver microsomes was performed in a $1.0 \mathrm{~mL}$ incubation volume containing $100 \mathrm{mM}$ sodium phosphate buffer $(\mathrm{pH} 7.6), 5 \mathrm{mM}$ magnesium chloride, $1 \mathrm{mM} \mathrm{NADP}{ }^{+}, 8 \mathrm{mM}$ glucose 6phosphate, 2 units glucose 6-phosphate dehydrogenase, 2 $\mathrm{mg}$ control-microsomes, and retrorsine $(2 \mu \mathrm{mol}$ in $50 \mu \mathrm{L}$ DMSO) at $37^{\circ} \mathrm{C}$ for $30 \mathrm{~min}$. After the incubation, the mixture was centrifuged at $105,000 \mathrm{~g}$ for $30 \mathrm{~min}$ at $4{ }^{\circ} \mathrm{C}$ to remove microsomal proteins. The supernatant fraction was collected and the resulting metabolite mixture was separated by reversed-phase HPLC employing two columns, a sample trap column (ODS, $4.6 \times 30 \mathrm{~mm}$ ) and an analytical column (Prodigy $5 \mu$ ODS, 4.6 x $250 \mathrm{~mm}$, Phenomenex, Torrance, CA). A switching valve was equipped between the sample trap and analytical columns. The sample was first loaded on to the sample trap column and washed with $20 \mathrm{mM}$ ammonium acetate buffer (buffer A) at the flow rate of 1 $\mathrm{mL} / \mathrm{min}$, so that the aqueous soluble impurities were directed to a waste bottle. After 5 min the sample trap column was switched to connect the analytical columns and the analysis was performed by eluting with linear gradient of buffer A to $50 \%$ methanol in buffer A (buffer B) over 30 min followed by isocratic elution with buffer B for $25 \mathrm{~min}$.

Metabolism of retrorsine by control or dexamethasone-induced microsomes from rat lung, kidney or spleen was similarly conducted, and the metabolites were similarly analyzed by HPLC. For enzyme inhibition study, metabolism of retrorsine was conducted in the presence of $100 \mu \mathrm{M}$ TAO.

\section{Preparation of DHP-Modified Calf thymus DNA}

Purified calf thymus DNA $(2.5 \mathrm{mg}, 7.5 \mu \mathrm{mol})$ in 2.5 $\mathrm{mL}$ of $20 \mathrm{mM} \mathrm{K}_{2} \mathrm{CO}_{3}(\mathrm{pH} \mathrm{7.5)}$ was reacted with $64 \mathrm{nmol}$ of DHP at $37{ }^{\circ} \mathrm{C}$ for $40 \mathrm{~min}$. After incubation, the reaction mixture was extracted twice with $2.5 \mathrm{~mL}$ of a chloroform/isoamyl alcohol mixture $(24 / 1, \mathrm{v} / \mathrm{v})$. The DNA in aqueous phase was precipitated by adding 250 $\mu \mathrm{L}$ of $3 \mathrm{M}$ sodium acetate followed by an equal volume of cold 2-propanol and washed with $70 \%$ ethanol. After the DNA was redissolved in $20 \mathrm{mM} \mathrm{K}_{2} \mathrm{CO}_{3}(\mathrm{pH} 7.5)$, the DNA concentration and purity were analyzed spectrophotometrically. The DNA was stored at $-78{ }^{\circ} \mathrm{C}$ prior to ${ }^{32} \mathrm{P}$-postlabeling/HPLC analysis.

\section{Metabolism of Retrorsine in the Presence of Calf Thymus DNA}

The metabolism of retrorsine in the presence of calf thymus DNA $(2.0 \mathrm{mg})$ was conducted in a $2 \mathrm{~mL}$ incubation volume with conditions similar to those for metabolism. After incubation, the reaction mixture was ultracentrifuged at $105,000 \mathrm{~g}$ for $30 \mathrm{~min}$ to remove the microsomal proteins. The clear supernatant was extracted twice with $2 \mathrm{ml}$ of chloroform/isoamyl alcohol 
(v/v, 24/1). The DNA in the aqueous phase was precipitated and purified as described above.

\section{Liver DNA Isolation}

Liver DNA of rats gavaged with retrorsine or with only phosphate buffer was extracted using RecoverEase DNA Isolation Kit (Stratagene, Cedar Creek, TX) according to the manufacturer's instructions. The concentration and purity of DNA isolated from rat liver were analyzed spectrophotometrically. ${ }^{32} \mathrm{P}-$ Postlabeling/HPLC analyses of DHP-derived DNA adducts ${ }^{32 \mathrm{P}}$-Postlabeling/HPLC analysis was conducted as described previously $(13,14)$. For quantitation of each sample, the two epimeric DHP-3'-dGMP synthetic standards, in an amount that closely matched the range of modification in the liver DNA samples, were also analyzed in parallel. Statistical comparisons were conducted by analysis of variance by Student's $t$-test.

\section{Results}

In vitro Metabolism of retrorsine by liver microsomes Figure 1 shows the chromatographic profile of reversedphase HPLC analysis of the in vitro retrorsine metabolism. The chromatographic peak that eluted at 43.6 min contained the recovered substrate, retrorsine. By comparison of HPLC retention times and UV-visible absorption with those of the DHP and retrorsine N-oxide standards $(14,19)$, the metabolites contained in chromatographic peaks eluting at 25.8 and $34.5 \mathrm{~min}$ were identified as DHP and retrorsine $N$-oxide, respectively (Figure 1).

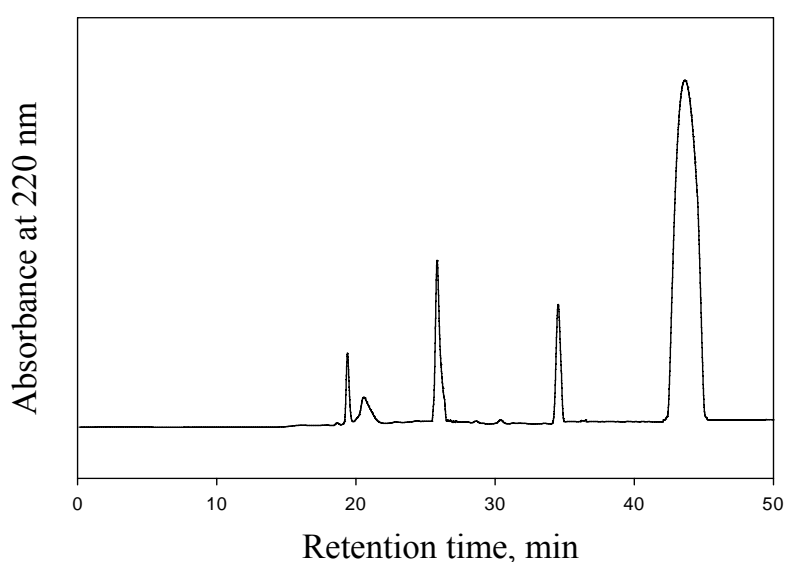

Figure 1: Reversed-phased HPLC analysis of metabolites formed from metabolism of retrorsine. For the conditions for HPLC analysis see Materials and Methods.

Since the chromatographic peaks eluted prior to 22 min were also detected from the incubation with liver microsomes pre-heated for $10 \mathrm{~min}$, these chromatographic peaks did not contain metabolites of retrorsine. Similar HPLC profiles of the metabolism of retrorsine by liver microsomes of rats treated with dexamethasone conducted under similar conditions were also obtained. The rate of DHP formation from metabolism of retrorsine by dexamethasone -microsomes was about 1.7-fold higher than that by controlmicrosomes (Table 1).

Table 1: Quantification of DHP and PA $N$-oxide formation in an in vitro PA metabolism by rat liver microsomes

\begin{tabular}{|c|c|c|c|c|}
\hline \multirow[b]{2}{*}{ Substrate } & \multicolumn{2}{|c|}{ Control-microsomes } & \multicolumn{2}{|c|}{$\begin{array}{c}\text { Dexamethasone- } \\
\text { microsomes }\end{array}$} \\
\hline & $\begin{array}{c}\text { DHP } \\
\text { (nmol/mg } \\
\text { /min) }\end{array}$ & $\begin{array}{c}N \text {-oxide } \\
\text { (nmol/mg } \\
\text { /min) }\end{array}$ & $\begin{array}{c}D H P \\
(\mathrm{nmol} / \mathrm{mg} \\
/ \mathrm{min})\end{array}$ & $\begin{array}{c}N \text {-oxide } \\
\text { (nmol/mg } \\
\text { /min) }\end{array}$ \\
\hline Retrors & $4.8 \pm 0.1^{1,2}$ & $17.6 \pm 0.5^{1,3}$ & $8.3 \pm 0.2^{1,2}$ & $25.1 \pm 0.9^{1,3}$ \\
\hline $\begin{array}{l}\text { Retrorsine } \\
+ \text { TAO }\end{array}$ & $1.1 \pm 0.04^{1}$ & $12.4 \pm 0.6^{1}$ & $2.7 \pm 0.7^{1}$ & $17.7 \pm 0.9^{1}$ \\
\hline \multicolumn{5}{|c|}{$\begin{array}{l}\text { Data represent the mean } \pm \mathrm{SD}(\mathrm{n}=3) \text {. For experimental details, } \\
\text { see Materials and Methods. } \\
{ }^{1} \text { Statistically significant difference }(\mathrm{p}<0.01) \text { between groups in } \\
\text { the same column. } \\
{ }^{2,3} \text { Statistically significant difference }(\mathrm{p}<0.05) \text { between groups } \\
\text { in the same row. }\end{array}$} \\
\hline
\end{tabular}

To determine whether or not P450 3A is the principal metabolizing enzyme that catalyzes metabolism of retrorsine to DHP and retrorsine $N$-oxide, metabolism of retrorsine by rat liver control and dexamethasonemicrosomes was conducted in the presence TAO, a specific P450 3A inhibitor. It was found that DHP formation were 77 and $67 \%$ reduced, respectively, compared with the metabolism by control and dexamethasone-induced microsomes without TAO (Table 1). Retrorsine $\mathrm{N}$-oxide formation in control and dexamethasone-microsomal metabolism was reduced by 30 and $29 \%$.

\section{In Vitro Metabolism by Extrahepatic Tissue Microsomes}

The quantifications of DHP and retrorsine $N$-oxide from the in vitro metabolism of retrorsine mediated by the microsomal fractions of extrahepatic tissues, lung, kidney and spleen of rats treated with dexamethasone are shown in Table 2. Compared to the retrorsine metabolism by liver microsomes from control rats, the retrorsine metabolizing enzyme activities in the extrahepatic tissues from dexamethasone-induced rats were much lower; the DHP and $\mathrm{N}$-oxide formation by the microsomal fractions obtained from the extrahepatic tissues of control rats were even lower (data not shown).

Table 2: Quantification of DHP and retrorsine N-oxide formation in an in vitro PA metabolism with lung, kidney, and spleen microsomes from the rats pretreated with dexamethasone

\begin{tabular}{lcc}
\hline Microsomes & $\begin{array}{c}\mathrm{DHP} \\
(\mathrm{pmol} / \mathrm{mg} / \mathrm{min})\end{array}$ & $\begin{array}{c}\text { Retrorsine N-oxide } \\
(\mathrm{pmol} / \mathrm{mg} / \mathrm{min})\end{array}$ \\
\hline Lung & $510 \pm 40$ & $727 \pm 26$ \\
Kidney & $79 \pm 3$ & $627 \pm 6$ \\
Spleen & $128 \pm 6$ & $702 \pm 39$ \\
\hline
\end{tabular}

Data represent the mean $\pm \mathrm{SD}(\mathrm{n}=3)$.

For experimental details, see Materials and Methods. 


\section{DHP-Derived DNA Adduct Formation}

Rat liver microsomal metabolism of retrorsine in the presence of calf thymus DNA was assayed and the resulting DNA adducts were analyzed by ${ }^{32} \mathrm{P}-$ postlabeling/HPLC. Metabolism of riddelliine in the presence of calf thymus DNA was performed in parallel to determine DNA adducts identification. As previously determined (13-15), female F344 rats fed riddelliine produced a set of eight DHP-derived DNA adducts in liver that were identical to the adduct peaks obtained from the ${ }^{32} \mathrm{P}$-postlabeling/HPLC analysis of DHPmodified calf thymus DNA (Figure 2A). These eight DHP-derived DNA adducts contained in the chromatographic peaks eluted at 47.6, 48.3, 51.4, 53.9, $55.3,60.1,61.0$, and $62.6 \mathrm{~min}$ are designated as P1, P2, P3, P4, P5, P6, P7, and P8, respectively. The DNA adducts designated as $\mathrm{P} 4$ and $\mathrm{P} 6$ are DHP-3'-dGMP adducts $(13,14)$ and the other six DHP-derived adducts (P1, P2, P3, P5, P7, and P8) were characterized as DHPderived dinucleotides (15). A similar HPLC profile was also obtained from the in vitro metabolism of retrorsine in the presence of calf thymus DNA (Figure 2B). Figure $2 \mathrm{C}$ shows the same eight DHP-derived DNA adducts formed from liver DNA of rats gavaged with retrorsine. The level of DNA adducts of retrorsine in liver of rats receiving three daily doses $(1 \mathrm{mg} / \mathrm{kg} /$ day $)$ is $110.3 \pm 18.0$ adducts $/ 10^{7}$ nucleotides.

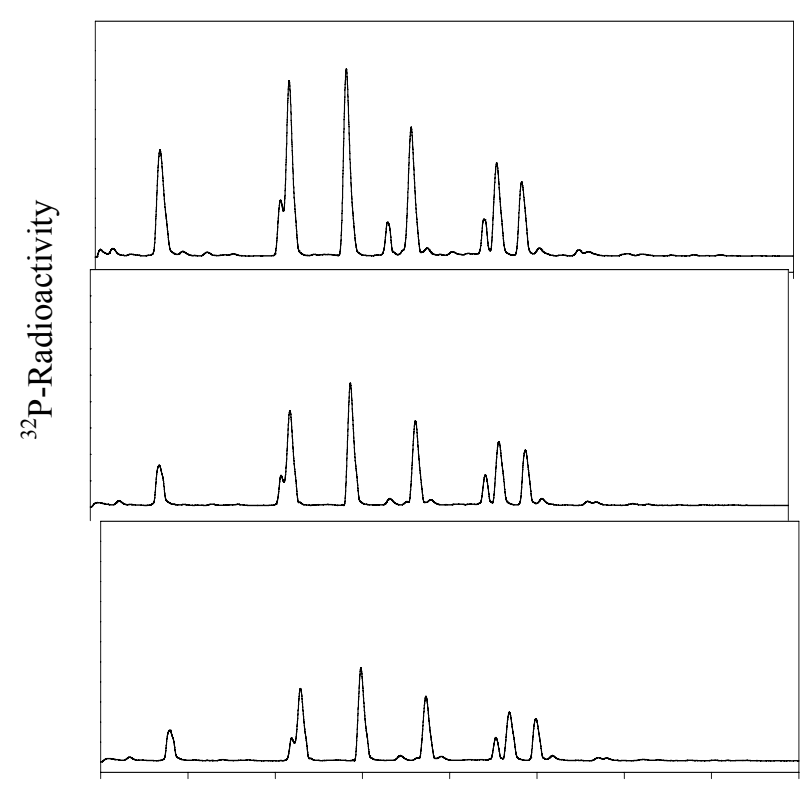

Retention time, $\min$

Figure 2: ${ }^{32}$ P-Postlabeling/HPLC analysis of DHPderived DNA adducts formed from (A) DHP-modified calf thymus DNA, (B) calf thymus DNA incubated with the in vitro metabolism of retrorsine, and (C) liver DNA of rats gavaged with retrorsine. The eight chromatographic peaks eluted at 47.6, 48.3, 51.4, 53.9, $55.3,60.1,61.0$, and $62.6 \mathrm{~min}$ are the identified DHPderived DNA adducts designated as P1, P2, P3, P4, P5, $\mathrm{P} 6, \mathrm{P} 7$ and P8, respectively. For the conditions for ${ }^{32} \mathrm{P}-$ postlabeling/HPLC analysis see Materials and Methods.

\section{Discussion}

Retrorsine exhibits a variety of toxic responses, including acute toxicity, mutagenicity, DNA crosslinking in cultured bovine kidney epithelial cells in the presence of an external metabolizing system (20), and clastogenic activity (21). Metabolism of retrorsine in vitro and in vivo formed isatinecic acid, pyrrolic metabolites, retrorsine $\mathrm{N}$-oxide, and retronecine $(17,22$, 23). Similar to riddelline, retrorsine and retrorsinecontaining plants induced liver tumors in rats (24).

CYP 3A was found to be the major isozyme for metabolizing monocrotaline and senecionine $(25,26)$. The inhibition of the DHP formation in the metabolism of retrorsine by TAO (Table 1) indicates that the metabolic formation of DHP was primarily catalyzed by CYP 3A enzyme. This finding is consistent with our previous report that metabolism of riddelliine by liver microsomes of female F344 rats is mainly catalyzed by CYP 3A. Xia et al. (27) performed a comparative study on the metabolism of riddelliine by human and rat liver microsomes and also found that the DHP and riddelliine $\mathrm{N}$-oxide were the major metabolites of riddelliine in an in vitro human microsomal incubation, with the levels comparable to those obtained from rat liver microsomal metabolism. Dexamethasone is not only a potent rat liver CYP3A inducer that induces 1.7 -fold rat liver retrorsine metabolizing enzyme activity but also induced microsomal retrorsine-metabolizing enzyme activity in lung, kidney and spleen. The CYP3A activity in the extrahepatic tissues of uninduced rats was very low; in fact, under our experimental conditions (2 mg microsomal protein, $30 \mathrm{~min}$ incubation) the DHP and retrorsine $\mathrm{N}$-oxide were hardly detected. However, using dexamethasone-induced lung, kidney, or spleen microsomes, we were able to determine the formation of DHP and retrorsine $N$-oxide. The levels of metabolism by extrahepatic tissue microsomes from the dexamethasone-induced rats were 18 to 32-fold lower than the metabolism of retrorsine by control liver microsomes.

We have previously found that the DHP-derived DNA adducts are formed in liver of male and female F344 rats fed riddelliine. The levels of the DHP-derived DNA adducts in liver DNA of rats treated with riddelliine correlated with the liver tumor incidence indicating the DHP-derived DNA adducts that are responsible for riddelliine-induced liver tumorigenicity (15); this riddelliine-induced tumorigenesis is mediated through a genotoxic mechanism. The current study shows the similar set of DHP-derived DNA adducts that was found in the liver DNA of rats treated with retrorsine and indicates the retrorsine-induced tumorigenesis may be also due to a genotoxic mechanism. The formation of these DNA adducts from retrorsine metabolism suggests that these DHP-derived DNA adducts are responsible for retrorsine-induced liver tumorigenicity as well as the other genotoxicities. Based on the results reported in this study and published previously (13), these DHP-derived DNA adducts are potential biomarkers of pyrrolizidine alkaloid-induced tumorigenicity. 
Based on the present findings, we propose that the metabolic activation of retrorsine leads to the formation of DHP-derived DNA adducts and liver tumors (Figure 3). The pyrrolic metabolite, dehydroretrorsine, can (i) bind to cellular DNA followed with hydrolysis to form the DHP-derived DNA adducts, or (ii) be hydrolyzed to form DHP followed by reaction with DNA to form the DHP-derived DNA adducts (Figure 3). Our results show that DHP is the common reactive metabolite generated by the retronecine-type pyrrolizidine alkaloids. Consequently, the formation of DHP-derived DNA adducts may well be important biomarkers for exposure to pyrrolizidine alkaloids.

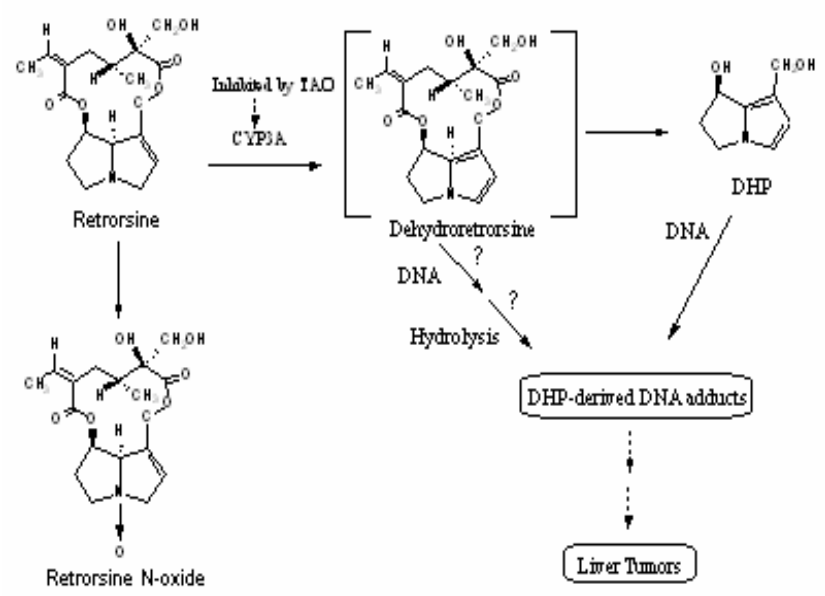

Figure 3: The proposed metabolic activation and detoxification pathways of retrorsine.

Acknowledgement: This research was supported in part by appointment (Y.W.) to the Postgraduate Research Program at the NCTR administered by the Oak Ridge Institute for Science and Education through an interagency agreement between the U.S. Department of Energy and the FDA.

\section{References}

1. Bull, L. B.; Culvenor, C. C.; Dick, A. J.: The pyrrolizidine alkaloids, their chemistry, pathogenicity and other biological properties. NorthHolland, Amsterdam, 1968.

2. International Agency for Research in Cancer (IARC): Pyrrolizidine alkaloids. In IARC Monograph on the evaluation of carcinogenic risk of chemicals to man - Some naturally occurring substance, International Agency for Research in Cancer: Lyon, France, 1976.

3. Mattocks, A. R.: Chemistry and Toxicology of Pyrrolizidine Alkaloids, London, New York: Academic Press, 1986.

4. Woo Y. T.; Lai, D. Y.; Arcos, J. C.; Argus, M. F.: Chemical Induction of Cancer. Academic Press Inc.: San Diego, 1988.

5. International Programme on Chemical Safety (IPCS): Pyrrolizidine Alkaloids Health and Safety Guide. Health and Safety Criteria Guide 26. WHO: Geneva. 1989.

6. Roeder, E.: Medicinal Plans in Europe Containing Pyrrolizidine Alkaloids. Pharmazie, 1995, 50 (2), 83-98.
7. Stegelmeier, B. L.; Edgar, J. A.; Colegate, S. M.; Gardner, D. R.; Schoch, T. K.; Coulombe Jr., R. A.; Molyneux, R. J.: Pyrrolizidine Alkaloid Plants, Metabolism and Toxicity. J. Nat. Toxins, 1999, 8 (1), 95-116.

8. Coulombe Jr., R. A.; Drew, G. L.; Stermitz, F. R.: Pyrrolizidine Alkaloids Crosslink DNA with Actin. Toxicol. Appl. Pharmacol., 1999, 154, 198-202.

9. Steenkamp, V.; Stewart, M. J.; Zuckerman, M.: Clinical and Analytical Aspects of Pyrrolizidine Poisoning Caused by South African Traditional Medicine. Ther. Drug Monit. 2000, 22 (3), 302-306.

10. Fu, P. P.; Chou, M. W.; Xia, Q.; Yang, Y. C.; Yan, J.; Doerge, D. R.; Chan. P. C.: Genotoxic Pyrrolizidine Alkaloids and Pyrrolizidine Alkaloid N-oxides-Mechanism Leading to DNA Adduct Formation and Tumorgenicity. Environ. Carcinogen Ecotoxicol. Rev., 2001, 19, 353-385.

11. Smith, L. W.; Culvenor, C. C.: Plant Sources of Hepatotoxic Pyrrolizidine Alkaloids. J. Nat. Prod., 1981, 44 (2), 129-152.

12. Chan. P. C.: NTP Technical Report on Toxicology and Carcinogenesis Studies of Riddelliine in F344/N

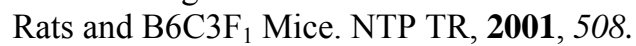

13. Yang, Y. C.; Yan, J.; Doerge, D. R.; Chan, P. C.; Fu, P. P.; Chou, M. W.: Metabolic Activation of the Tumorgenic Pyrrolizidine Alkaloid, Riddelliine, Leading to DNA adduct Formation in Vivo. Chem. Res. Toxicol., 2001, 14 (1), 101-109.

14. Yang, Y. C.; Yan, J.; Churchwell, M.; Beger, R.; Chan, P.; Doerge, D. R.; Fu, P. P.; Chou, M. W.: Development of ${ }^{32}$ P-Postlabeling/ HPLC method for Detection of Dehydroretronecine-Derived DNA Adduct in Vitro and in Vivo. Chem. Res. Toxicol., 2001, 14 (1), 91-100.

15. Chou, M. W.; Yan, J.; Williams, L.; Xia, Q.; Churchwell, M.; Doerge, D. R.; Fu, P. P.: Identification of DNA Adduct Derived from Riddelliine, a Carcinogenic Pyrrolizidine Alkaloid. Chem. Res. Toxicol., 2003, 16(9), 1130-1137.

16. Chou, M. W.; Yan, J.; Nichols, J.; Xia, Q.; Beland, F. A.; Chan, P. C.; Fu, P. P.: Correlation of DNA Adduct Formation and Riddelliine-induced Liver Tumorigenesis in F344 Rats and B6C3F $F_{1}$ Mice. Cancer Lett, 2003, 193 (2), 119-125.

17. Chu, P. S.; Lame, M. W; Segall. H. J.: In Vivo Metabolism of Retrorsine and Retrorsine-N-oxide. Arch Toxicol, 1993, 67 (1), 39-43.

18. Mattocks, A. R.; White, I. N.: Toxic Effects and Pyrrolic Metabolites in the Liver of Young Rats Given the Pyrrolizidine Alkaloid Retrorsine. ChemBiol. Interactions, 1973, 6 (5), 297-306.

19. Chou, M. W.; Wang, Y. P.; Yan, J.; Yang; Y. C.; Beger, R.; Williams, L. D.; Doerge, D. R.: Fu. P. P.: Riddelliine N-oxide is a Photochemical and Mammalian Metabolite with Genotoxic Activity That is Comparable to the Parent Pyrrolizidine Alkaloid Riddelliine. Toxicol. Lett., 2003, 145 (3), 239-247.

20. Hincks, J. R.; Kim, H. Y.; Segall, H. J.; Molyneux, R. J.; Stermitz, F. R.; Coulombe Jr., R. A.: DNA Cross-linking in Mammalian Cells by Pyrrolizidine Alkaloids, Structure-Activity Relationships. Toxicol Appl Pharmacol, 1991, 111 (1), 90-98. 
21. Kedzierskiand B.; Buhler. D. R.: The Formation of 6,7-dihydro-7-hydroxy-1-hydroxymethyl-5Hpyrrolizine, a Metabolite of Pyrrolizidine Alkaloids. Anal Biochem., 1986, 152 (1), 59-65.

22. Chan, M. Y.; Zhao, X. L.; Ogle, C. W.: A Comparative Study on the Hepatic Toxicity and Metabolism of Crotalaria Assamica and Eupatorium Species. Am J Chin Med., 1989, 17 (3-4), 165-170.

23. Couet, C. E.; Hopley, J.; Hanley, A. B.: Metabolic Activation of Pyrrolizdine Alkaloids by Human, Rat and Avocado Microsomes. Toxicon., 1996, 34(9), 1058-1061.

24. Schoental, R.; Head, M. A.; Peacock, P. R.: Senecio Alkaloids: a Primary Liver Tumours in Rats as a Results of Treatment with (1) A Mixture of Alkaloids from S. Jakabae Lin; (2) Retrorsine; (3) Isatidine. Br. J. Cancer, 1954, 8 (3), 458-465.
25. Reid, M. J.; Lame, M. W.; Morin, D.; Wilson, D. W. Segall, H. J.: Involvement of Cytochrome P450 3A in the Metabolism and Covalent Binding of ${ }^{14} \mathrm{C}$ Monocrotaline in Rats Liver Microsomes. $J$. Biochem Mol Toxicol., 1998, 12 (3), 157-166.

26. Chung, W. G.; Buhler, D. R.: The Effect of Spironolacton Treatment on the Cytochome P450mediated Metabolism of the Pyrrolizidine Alkaloid Senecionine by Hepatic Microsomes from Rats and Guinea Pigs. Toxicol. Appl. Pharmacol., 1994, 127(2), 314-319.

27. Xia Q.; Chou, M. W.; Kadlubar, F. F.; Chan, P. C. Fu, P. P.: Human Liver Microsomal Metabolism and DNA Adduct Formation of Tumorigenic Pyrrolizidine Alkaloid, Riddelliine. Chem Res Toxicol., 2003, 16 (1), 66-73. 